Please do not remove this page

RMIT

UNIVERSITY

\title{
Time-frequency analysis of normal and abnormal biological signals
}

Mahmoud, Seedahmed; Hussain, Zahir; Cosic, Irena; Fang, John

https://researchrepository.rmit.edu.au/esploro/outputs/9921862883201341/filesAndLinks?institution=61RMIT_INST\&index=null

Mahmoud, S., Hussain, Z., Cosic, I., \& Fang, J. (2006). Time-frequency analysis of normal and abnormal biological signals. Biomedical Signal Processing and Control, 1(1), 33-43.

https://doi.org/10.1016/j.bspc.2006.02.001

Published Version: https://doi.org/10.1016/j.bspc.2006.02.001

Repository homepage: https://researchrepository.rmit.edu.au

Copyright (c) 2006 Elsevier Ltd All rights reserved.

Downloaded On 2023/04/26 22:07:02 +1000

Please do not remove this page 


\section{Time-Frequency Analysis of Normal and Abnormal Biological Signals}

\section{Seedahmed S. Mahmoud, Zahir M. Hussain, Irena Cosic, and Qiang Fang}

School of Electrical and Computer Engineering, RMIT, Melbourne, Victoria 3000, Australia. E-mail addresses:

mahmoud.seedahmed@rmit.edu.au,zahir.hussain@rmit.edu.au,irena.cosic@rmit.edu.au, john.fang@rmit.edu.au

Corresponding Author:

Dr. Qiang Fang

School of Electrical and Computer Engineering

RMIT, 124 Latrobe Street, Melbourne, Victoria 3000, AUSTRALIA

Tel. : +61 3 99252432, Fax. +61-3-9925-2007

E-mail: john.fang@rmit.edu.au

Citation:

Mahmoud, S, Hussain, Z, Cosic, I and Fang, J 2006, 'Time-frequency analysis of normal and abnormal biological signals', Biomedical Signal Processing and Control, vol. 1, no. 1, pp. 33-43. 


\begin{abstract}
Due to the non-stationary, multicomponent nature of biomedical signals, the use of timefrequency analysis can be inevitable for these signals. The choice of the proper time-frequency distribution (TFD) that can reveal the exact multicomponent structure of biological signals is vital in many applications, including the diagnosis of medical abnormalities. In this paper, the instantaneous frequency (IF) estimation using four well-known TFDs is applied for analyzing biological signals. These TFDs are: the Wigner-Ville distribution (WVD), the Choi-Williams distribution (CWD), the Exponential T-distribution (ETD) and the Hyperbolic T-distribution (HTD). Their performance over normal and abnormal biological signals as well as over multicomponent frequency modulation (FM) signals in additive Gaussian noise was compared. Moreover, the feasibility of utilizing the wavelet transform (WT) in IF estimation is also studied. The biological signals considered in this work are the surface electromyogram (SEMG) with the presence of ECG noise and abnormal cardiac signals. The abnormal cardiac signals were taken from a patient with malignant ventricular arrhythmia, and a patient with supraventricular arrhythmia. Simulation results showed that the HTD has a superior performance, in terms of resolution and cross-terms reduction, as compared to other time-frequency distributions.
\end{abstract}

\title{
Key Words
}

Biological signal, ECG, EMG, time-frequency analysis. 


\section{INTRODUCTION}

Traditionally, biological signals such as electrocardiogram (ECG), electroencephalogram (EEG) and electromyogram (EMG) are analyzed in the time-domain by skilled physicians. However, pathological conditions may not always be obvious in the time-domain signal. For example, in a subject with arrhythmia or a subject undergoing an epileptic seizure, certain rhythms become more prominent where they experience change in amplitude and frequency. Investigation of such biological signals can assist the surgeon in deciding on surgical intervention [1]. Sometimes, biological signals accompany by noise that may consist of artefact or environmental interference. These facts have motivated the use of frequency domain techniques, such as Fourier transform (FT), for analysis [2]. However, as the ECG and all other biological signals belong to the family of multicomponent nonstationary signals [3], accurate time-varying spectral estimates can be extremely difficult to obtain. However, a proper time-frequency distribution (TFD) can tackle this problem and reveal the multicomponent nature of such signals.

Time-frequency analysis plays a significant role in signal processing and biomedical engineering $[4,5]$. The instantaneous frequency (IF) is an important concept in time-frequency analysis, especially when analyzing multicomponent signals. The concept of the instantaneous frequency can be found in $[6,4,5,7]$. Every TFD has a ridge or concentration of energy in the timefrequency plane around the instantaneous frequency (IF) of each component. As such it can be used to estimate the frequency variation of the ECG (or any biomedical) signal over time. This presentation provides information where the time-domain and frequency-domain may fail to produce. In particular, it uses to detect the QRS complex and arrhythmia [8]. Methods of IF estimation can be classified into two major categories: parametric and non-parametric. Parametric IF estimation methods are complicated and time-consuming, hence not suitable for real-time applications. Non-parametric IF estimation for multicomponent nonstationary signals is an important (and unresolved) issue in signal processing [6,9]. Although Fourier analysis can reveal the multicomponent nature of signals in some special cases (e.g., sum of sinusoids), it is only time-frequency analysis that can be used for general IF estimation for multicomponent signals through concentrating the signal energy in the time-frequency plane around the component IF laws [4]. There are many TFDs in active use, the most significant class of TFDs is known as the Quadratic Class or Cohen's Class [4]. However, quadratic time - frequency distributions suffer from the presence of cross-terms when used to analyze multicomponent signals $[4,5,7]$. Cross-terms are fictitious concentrations of energy, resulting from the quadratic nature of Cohen's class, which 
can obscure the real features of interest in the signal. On the other hand, joint time-frequency resolution is another significant character that is different for different TFDs. In some applications (e.g., biomedical signal analysis), we may be confronted by multiple components with narrow separation in time, frequency, or both; in such a case many TFDs fail to reveal the true structure as many components will overlap due to bad resolution. Considerable efforts have been made to define TFDs which reduce the effect of cross-terms while improving the time-frequency resolution $[4,7]$. However, there is always a compromise between these two requirements. TFD's have different performances in this respect and the choice of the proper TFD is application dependent.

This paper is organized as follows. In Section II we will explain the process of IF estimation, both in principle and numerically. In Section III, the acquisition of normal and abnormal biological signals is explained. In Section IV we will discuss four time-frequency distributions (TFDs) that will be used for the comparison purposes in this paper. Two of these TFDs are classified as timeonly kernels distributions. Section V discusses the process of frequency estimation using this class, the T-distribution. Extensive performance comparison of the four TFDs over biological signals and noisy mono- and multicomponent FM signals will be presented in Section VI.

\section{INSTANTANEOUS FREQUENCY ESTIMATION}

Consider a real signal $s(t)$. To avoid aliasing in the digital implementation of the TFD for this signal, we always consider its analytic associate $z(t)=s(t)+j \hat{s}(t)$, where $\hat{s}(t)$ is the Hilbert transform of $s(t)[5]$.

Biological signals such as EEG can be described as a nonstationary random signal composed of an amplitude modulation-frequency modulation (AM-FM) part in additive stationary random noise, with low signal-to-noise ratio (SNR) [10]. As the AM variation is normally slow without sudden or abrupt changes, noisy multicomponent IF estimation techniques are applicable to biomedical signals. To verify the concept of IF we consider an analytic FM signal of the form

$$
z(t)=a e^{j \phi(t)}+\epsilon(t)
$$

where the amplitude $a$ is constant, $\phi(t)$ is the phase of the analytic signal, and $\epsilon(t)$ is a complexvalued white Gaussian noise with independent identically distributed (i.i.d.) real and imaginary parts with total variance $\sigma_{\epsilon}^{2}$. The instantaneous frequency of $z(t)$ is given by the derivative of the phase as follows:

$$
f_{i}(t)=\frac{1}{2 \pi} \frac{d \phi(t)}{d t}
$$


We assume that $f_{i}(t)$ is an arbitrary, smooth and differentiable function of time with bounded derivatives of all orders.

The continuous time-frequency distribution of the analytic signal $z(t)$ associated with the original real signal $s(t)$ can be expressed as follows $[5,6]$

$$
\rho(t, f)=\underset{\tau \rightarrow f}{\mathcal{F}}\left[G(t, \tau) \underset{(t)}{*} K_{z}(t, \tau)\right]
$$

where $K_{z}(t, \tau)=z(t+\tau / 2) z^{*}(t-\tau / 2)$ is the instantaneous autocorrelation product, $\mathcal{F}$ is the Fourier transform, $G(t, \tau)$ is the time-lag kernel, and $\underset{(t)}{*}$ denotes time convolution. It is wellknown that the kernel can completely characterize the TFD and its properties (e.g., resolution) [12]. The kernel can also be expressed in the Doppler-lag domain as follows

$$
G(t, \tau)=\underset{\nu \rightarrow t}{\mathcal{F}^{-1}}\{g(\nu, \tau)\}
$$

For practical implementation we need the discrete version of the TFD. First, in the discrete lag-domain, the TFD $\rho(t, f)$ can be expressed as follows

$$
\rho(t, f)=\int_{-\infty}^{\infty} \sum_{m=-\infty}^{\infty} K_{z}(u, 2 m T) G(t-u, 2 m T) e^{-j 4 \pi f m T} d u
$$

where $m$ is an integer and $T$ is the sampling interval. Second, if $\rho(t, f)$ is discretized over time and frequency we get

$$
\rho(n, k)=\sum_{l=-N}^{N-1} \sum_{m=-N}^{N-1} K_{z}(l T, 2 m T) G(n T-l T, 2 m T) e^{-j 2 \pi \frac{k m}{2 N}}
$$

where $2 N$ is the total number of signal samples. The implementation discrete frequency is given by $f_{k}=k / 4 N T$. Since all TFDs has a peak or a ridge around the IF, then the IF estimate will be a solution of the following optimization problem:

$$
\widehat{f}_{i}(t)=\arg \left[\max _{f} \rho(t, f)\right] ; 0 \leq f \leq f_{s} / 2
$$

where $f_{s}=1 / T$ is the sampling frequency. The frequency estimation error is the difference between the actual value in eq.(2) and the estimate in eq.(7) as follows:

$$
\Delta \widehat{f}_{i}(t)=f_{i}(t)-\widehat{f}_{i}(t)=\phi^{\prime}(t) / 2 \pi-\widehat{f}_{i}(t)
$$


The most important factors that decide the quality of estimation are the bias and the variance of the estimate. In the above IF estimate, the bias and variance can be described as follows:

$$
\begin{aligned}
B\left(\hat{f}_{i}(t)\right) & =\mathcal{E}\left[\Delta \hat{f}_{i}(t)\right]=f_{i}(t)-\mathcal{E}\left[\hat{f}_{i}(t)\right] \\
V\left(\hat{f}_{i}(t)\right) & =\mathcal{E}\left[\Delta \hat{f}_{i}(t)\right]^{2}=\mathcal{E}\left[\left\{f_{i}(t)-\hat{f}_{i}(t)\right\}^{2}\right] .
\end{aligned}
$$

For the T-Class of TFD's, this bias is zero for single-tone and linear FM (LFM) signals, and therefore a Cramer-Rao bound (CRB) exists for the variance. For the ECG signals, no significant non-linearities in the component IF lags are expected, so we expect a very low bias (almost zero) and a small variance that is not different from the LFM case.

\section{ACQUISITION OF BIOLOGICAL SIGNALS}

In this comparative study we consider two types of biological signals. These signals are:

1. Surface electromyogram (SEMG) with the presence of ECG noise (normal signal with artifacts) and,

2. Abnormal cardiac signals, ECG, for a patient with malignant ventricular arrhythmia, and a patient with supraventricular arrhythmia (these arrhythmia data were obtained from the Physionet database [13]).

Utilization of time-frequency analysis as a preprocessing stage is extremely important for both signals. For the SEMG contaminated with an ECG, IF estimation of such a signal will reveal all of its structural components and clearly provide the frequency components of the ECG signal (P-QRS-T signature) as well as their time of occurrence. This will help in the removal of these artifacts from the SEMG.

Due to its high time-frequency resolution and cross-terms control, the T-distributions are expected to be efficient in analyzing more delicate biological signals like the EEG signal. TFD ridge analysis for EEG signals appeared in [14]. The problem with cross-terms, resolution, and IF estimation is more critical in EEG signals than in others, as the EEG signal may have very close time-frequency components (ridges).

\subsection{Surface Electromyogram}

Surface electromyogram (SEMG) is a result of a noninvasive recording-technique of the electrical activity of skeletal muscles [15]. It can be used to measure the properties of muscles supporting 
spinal segments. It is stochastic in nature and can be represented by a Gaussian distribution function [16]. However, the recording of such a signal suffers from the presence of an ECG signal [17].

In this measurement, a pair of surface-EMG electrodes were positioned on the right side of the spine of the subject. The ground electrode was placed on the spinal cord [18]. The subject was sitting normal (SitN). These electrodes were connected to BIOPAC systems, EMG module. The EMG module (EMG 100C) consists of instrumentation amplifier (IA) and a $50 \mathrm{~Hz}$ notch filter. The IA gain was set to 1000. The ECG data were recorded by using AcqKnowledge software (v.3.7.1, BIOPAC Systems, Inc., CA) in ASCII text files and processed by programs written in Matlab. The sampling rate for EMG was set to 1000 samples/second and down-sampled to 200 samples/second for time frequency analysis for memory limitation.

Fig. (1) shows the time domain of the raw SEMG signal with the ECG artefact during sitting normal posture.

Figure 1: Time-domain signal of the raw SEMG with ECG artefact during SitN (sampling frequency = 200 sample/seccond).

\subsection{Abnormal Electrocardiogram}

The electrocardiogram (ECG) signal has a well-defined P, QRS, T signature that represents with each heart beat. The P-wave arises from the depolarization of the atrium. The QRS complex arises from depolarization of the ventricles and T-wave arises from repolarization of the ventricle muscles. The duration, shape and amplitude of these waves are considered as major features in time domain analysis. Sometimes, the time morphologies of these waves are similar. The IF estimation of such signal using high resolution TFD can provide some parameters which behave differently than that of the time or frequency domain techniques.

An arrhythmia is an abnormality in the heart's rhythm, or heart beat pattern. The heart beat can be too slow, too fast, have extra beats, or otherwise beat irregularly [19]. The types of abnormal ECG signals investigated in this study were: supraventricular arrhythmia and malignant ventricular arrhythmia. Supraventricular arrhythmia occurs in the upper areas of the heart and is less serious than ventricular arrhythmia. It has irregular shapes of QRS complexes [19]. These arrhythmia data, supraventricular arrhythmia and malignant ventricular arrhythmia, were obtained from the Physionet database [13]. A Matlab program was used to convert the ECG binary format into Matlab format to be processed by the TFD. The sampling frequencies for supraventricular arrhythmia and malignant ventricular arrhythmia were 128 sample/secconds and 
250 sample/secconds, respectively.

Fig. (2) shows the time-domain ECG signal of a patient with supraventricular arrhythmia. The shape of the QRS complex in this signal is abnormal at the QR part. It requires high resolution TFD to detect the change that occurs in a narrow duration of time.

Fig. (3) shows the time-domain ECG signal of a patient with malignant ventricular arrhythmia. The depolarisation wave spreads through the ventricles by an irregular and therefore slower pathway. The QRS complex is thus wide and abnormal. Repolarisation pathways are also different, causing the $\mathrm{T}$ wave to have an unusual morphology. These sort of signals require a selection of an appropriate time-frequency distribution that is capable of handling multicomponent signals and tracking the abnormality in the signal.

Figure 2: Time-domain of signal-800 from supraventricular arrhythmia database (sampling frequency $=$ 128 sample/secconds).

Figure 3: Time-domain of signal-418 from malignant ventricular arrhythmia database (sampling frequency $=250$ sample $/$ secconds $)$.

\section{TIME-FREQUENCY ANALYSIS TECHNIQUES}

Time-frequency analysis allows a joint time - frequency resolution. However, when used for analyzing multicomponent signals, this approach is susceptible to cross-terms arising in the middle between the time-frequency (energy) components. Therefore, a TFD that provides a good reduction of the cross-terms is needed to make sure that the off-diagonal elements of the TFD matrix of the sources are negligible and that a diagonal structure can be maintained. In this study we investigate and compare the performance of four TFD's in analyzing biological signals. These distributions are

1. The Wigner - Ville distribution (WVD).

2. The Choi - Williams distribution (CWD).

3. The Exponential T-distribution (ETD).

4. The Hyperbolic T-distribution (HTD).

In the following subsections these distributions will be briefly described. 


\subsection{The Wigner-Ville distribution}

The Wigner - Ville distribution (WVD) of a continuous signal $z(t)$ is defined as [20]

$$
W V(t, f)=\int_{-\infty}^{\infty} z\left(t+\frac{\tau}{2}\right) z^{*}\left(t-\frac{\tau}{2}\right) e^{-j 2 \pi f \tau} d \tau
$$

where $f$ is the frequency variable. The WVD satisfies a large number of desirable mathematical properties. In particular, WVD is always real-valued, it preserves time and frequency shifts and satisfies the marginal properties. Based on (3), the WVD, which utilizes a time-only kernel $G(t, \tau)=\mathrm{G}(t)=\delta(t)$ with $g(\nu, \tau)=\mathrm{g}(\nu)=1$, has significant oscillatory cross-terms without a controlling factor, where the cross-terms can be larger in amplitude than the auto-terms. However, using a low-pass time-only kernel other than $\delta(t)$ will result in controlling the cross-terms by the low-pass function g [21].

\subsection{The Choi-Williams Distribution}

The Choi - Williams distribution $C W(t, f)$ was a significant step in the field of time-frequency analysis where it opened the way for optimizing resolution with cross-terms reduction [24]. The kernel of the Choi-Williams distribution (CWD) in the Doppler - lag domain is $(g(\nu, \tau)=$ $\left.\exp \left(-4 \pi^{2} \nu^{2} \tau^{2} / \sigma\right)\right)$ which can be given in the time-lag domain by [5]

$$
G(t, \tau)=\sqrt{\sigma / 4 \pi \tau^{2}} \exp \left(-\sigma t^{2} / 4 \tau^{2}\right)
$$

where $\sigma$ is a real parameter that can control the resolution and the cross-terms reduction. This two-dimensional exponential kernel has shown excellent performance in reducing cross-terms while keeping high resolution, with a compromise between these two requirements decided by the parameter $\sigma$.

\subsection{The Exponential T-distribution (ETD)}

The Exponential T-distribution (ETD) is a time-only kernel distribution. Time-only kernels are a special case of separable time-lag kernels. Suppose we have a separable time-lag kernel as follows

$$
G(t, \tau)=g_{1}(t) g_{2}(\tau)
$$

where $g_{1}$ and $g_{2}$ are continuous and $L^{2}$ integrable functions of time and lag, respectively. 
It was shown in [12] that for best time-frequency resolution we should have

$$
\begin{aligned}
& G(t, \tau)=R(t)=g_{1}(t) / M \\
& g(\nu, \tau)=r(\nu)=\underset{\tau \rightarrow v}{\mathcal{F}_{\tau \rightarrow v}^{-1}}\left\{g_{1}(t)\right\} / M
\end{aligned}
$$

where $M=\int g_{2}(u) d u$ is a constant and $G(t, \tau)$ is now a time-only kernel. This is the formula for all time-only kernels, which are the kernels of the T-distributions.

To examine the behavior of this kind of kernels in terms of resolution and cross-terms reduction, we consider a sum of two complex sinusoids

$$
z(t)=a_{1} \exp \left\{j\left(2 \pi f_{1} t+\theta_{1}\right)\right\}+a_{2} \exp \left\{j\left(2 \pi f_{2} t+\theta_{2}\right)\right\}
$$

where $a_{1}, a_{2}$ are real constants and $\theta_{1}$ and $\theta_{2}$ are phase constants. We obtain

$$
\begin{aligned}
\rho_{z}(t, f) & =a_{1}^{2} \delta\left(f-f_{1}\right)+a_{2}^{2} \delta\left(f-f_{2}\right)+2 a_{1} a_{2} g\left(f_{1}-f_{2}\right) \\
& \times \cos \left\{2 \pi\left(f_{1}-f_{2}\right) t+\theta_{1}-\theta_{2}\right\} \delta\left(f-\frac{f_{1}+f_{2}}{2}\right)
\end{aligned}
$$

where there is an ideal concentration about the auto-terms, and cross-terms appear with a controlling factor $\mathrm{g}\left(f_{1}-f_{2}\right)$ [21]. In case of two complex sinusoids above we have the controlling factor $\mathrm{g}\left(f_{1}-f_{2}\right)$ with cross-terms reduction that depends on the shape of the low-pass function $\mathrm{g}$ and the frequency separation $f_{1}-f_{2}$, where better cross-terms reduction is obtained for wider frequency separation.

A time-frequency distribution $T_{e}(t, f)$ with the exponential time-only kernel was proposed in [12], where the kernel was given by

$$
G(t, \tau)=R_{\sigma}(t)=\sqrt{\sigma / \pi} \exp \left(-\sigma t^{2}\right)
$$

$\sigma$ being a real parameter and $\sqrt{\sigma / \pi}$ is a normalization factor. It was shown in [6] that the resolution of the ETD exceeds that of CWD by far.

\subsection{The Hyperbolic T-distribution (HTD)}

The Hyperbolic T-distribution (HTD) is another time-only kernel distribution. The kernel 
(the hyperbolic time-only kernel) for this distribution is given by [12]

$$
G(t, \tau)=R_{\sigma}(t)=\frac{k_{\sigma}}{\cosh ^{2 \sigma}(t)}
$$

where $\sigma$ is a real positive number and $k_{\sigma}$ is a normalization factor given by

$$
k_{\sigma}=\int_{-\infty}^{\infty} \frac{1}{\cosh ^{2 \sigma}(t)} d t=\Gamma(2 \sigma) / 2^{2 \sigma-1} \Gamma^{2}(\sigma)
$$

in which $\Gamma$ represents the gamma function. In the following section we will discuss the IF estimation of the exponential and hyperbolic T-distributions. Their performance on linear and non-linear FM signals will be considered.

\section{WAVELET TRANSFORM}

Wavelets have found application in many aspects of biomedical signal processing such as feature extraction, noise reduction, data compression and QRS detection. The general equation of the wavelet transformation for a time signal $z(t)[22]$ is given as

$$
Q_{z}(\tau, a)=\frac{1}{\sqrt{a}} \int_{-\infty}^{\infty} z(t) g^{*}\left(\frac{t-\tau}{a}\right) d t
$$

where $\tau$ is the time shift parameter, $a$ is the dilation parameter (scale) which governs the frequency, and $g(t)$ is called the basic wavelet or mother wavelet. The mother wavelets must satisfy the admissibility condition [23]. There is a variety of mother wavelets that are application-dependent. For example, a widely used wavelet is the Morlet's wavelet, defined as

$$
g\left(\frac{t}{a}\right)=e^{j \frac{\omega_{o}}{a} t} e^{-\frac{t^{2}}{2 a^{2}}} .
$$

where $\omega_{o}$ is a constant.

The wavelet time-scale representation can also be viewed as a time- frequency one, where the analyzing frequency can be taken from the scale via $\omega_{o} / 2 \pi a, \omega_{o}$ being the bandwidth of the mother wavelet [22]. In this section we study the IF estimation of multicomponent non-linear FM signal using wavelet transform (WT). WT is a good tool for multi-resolution analysis due to its

"zoomable" resolution while the scale changes, however, it is not appropriate for IF estimation.

Fig. (4: up) shows that the Daubechies-10 WT (db10) of a mono-component non-linear FM 
signal given by $x_{1}(t)=\cos \left[2 \pi\left(f_{o} t+e t^{2}\right)\right]$ with $f_{o}=0.5 \mathrm{~Hz}$ and $e=0.5$. It is evident that we can take the IF of the signal from the ridge of the WT. However, the resolution will be different between high and low frequencies.

Fig. (4: down) shows the time-scale representation of a two-component nonlinear FM signal using the db10 WT. The two components are widely separated, where the instantaneous frequency of one component is five times the IF of the other as follows:

$$
x(t)=\cos \left[2 \pi\left(f_{o} t+e t^{2}\right)\right]+\cos \left[2 \pi\left(5 f_{o} t+e t^{2}\right)\right]
$$

with $f_{o}=0.5 \mathrm{~Hz}$ and $e=0.5$.

Fig. (5) displays the Morlet WT for the same signals as above. The difference in resolution from Fig. (4) is clear, however, both transforms share the cross-terms problem. The cross-terms is an inevitable problem that obscures IF estimation for multi-component signals using WT. Unlike the reduced interference TFDs [24], which can control the cross-terms using special design techniques, the WTs cannot control the cross-terms [25]. As we are considering multi-component signals in this paper, we will concentrate on IF estimation using TFDs.

Figure 4: Time-scale representations of a mono- and a two- component nonlinear FM signals using the db10 WT.

Figure 5: Time-scale representations of the same FM signals as in the previous figure using the Morlet WT.

\section{FREQUENCY ESTIMATION USING T-DISTRIBUTIONS}

It can be shown that the T-distributions do not satisfy the time marginal property, hence they do not satisfy the traditional condition for the instantaneous frequency. But in [6], Zahir et al proposed the following general IF property: at any time $t$, the time-frequency distribution $\rho_{z}(t, f)$ should have absolute maximum at $f=\frac{1}{2 \pi} \frac{d \phi(t)}{d t}$, which is the actual important characteristic needed for IF estimation. They have also shown that at any $t$, the hyperbolic T-distribution has

an absolute maximum at $f=\frac{1}{2 \pi} \frac{d \phi(t)}{d t}$ for linear FM signals. This is general for all T-distributions and constitutes the basis for our IF estimation. For non-linear FM signals this IF estimate is 
biased [6]. For an FM signal of the form $z(t)=a e^{j \phi(t)}, a$ being a constant, the general formula for the T-distributions can be given by

$$
\begin{aligned}
\rho_{z}(t, f) & \approx|a|^{2} \int R_{\sigma}(t-u) \delta\left[\frac{1}{2 \pi} \phi^{\prime}(u)-f\right] d u \\
& =|a|^{2} R_{\sigma}(t-\psi(f)) \psi^{\prime}(f)
\end{aligned}
$$

where $\psi$ is the inverse of $\frac{1}{2 \pi} \phi^{\prime}$, i.e., $\frac{1}{2 \pi} \phi^{\prime}(\psi(f))=f$ and it is assumed that there is a relatively small effect from higher-order derivatives $\phi^{(k)}(t), k \geq 3$. Assuming that $\psi^{\prime}(f)$ is not a highly peaked function of $f$ and knowing that $R_{\sigma}(t-\psi(f))$ is peaked at $t=\psi(f)$ since it is low-pass and even in $t$, the absolute maximum of $\rho_{z}(t, f)$ for any time $t$ would be at $\psi(f)=t$, or $f=\frac{1}{2 \pi} \phi^{\prime}(t)$, which is the instantaneous frequency of the FM signal $z(t)$. For non-linear FM signals, the energy peak of $\rho_{z}(t, f)$ is biased from the instantaneous frequency due to the higher-order phase derivatives. The major contribution in this term is due to $\phi^{(3)}(u)$ [6]. Therefore at the instants of rapid change in the IF law the bias is not negligible and eq.(16) would not be an accurate approximation to $\rho(t, f)$ without a suitable lag windowing. However, these abrupt changes are not expected in ECG signals (actually most biomedical signals). Hence, we needn't to consider non-linear IF law estimation techniques (like adaptive lag-window length as in [6]).

For linear FM (LFM) signals we have $\phi^{(k)}(t)=0$ for $k \geq 3$. If $\phi(t)=2 \pi\left(f_{o} t+\beta_{o} t^{2} / 2\right)$, where $f_{o}, \beta_{o}$ are constants, we have

$$
\rho_{z}(t, f)=\frac{1}{\beta_{o}}|a|^{2} R_{\sigma}\left[t-\frac{1}{\beta_{o}}\left(f-f_{o}\right)\right]
$$

which has an absolute maximum at $f=f_{o}+\beta_{o} t$, the instantaneous frequency. As $\beta_{o} \rightarrow 0$, the linear-FM signal $z(t)$ will approach a sinusoid, and we have $\rho(t, f) \rightarrow|a|^{2} \delta\left(f-f_{o}\right)$ for a monocomponent single-tone signal. For a signal composed of the sum of two LFM signals $z(t)=a_{1} e^{j \phi_{1}(t)}+a_{2} e^{j \phi_{2}(t)}$ with $\phi_{i}(t)=2 \pi\left(f_{i} t+\beta_{i} t^{2} / 2\right), i \in\{1,2\}$, the T-distribution can be expressed as follows:

$$
\rho_{z}(t, f)=\frac{1}{\beta_{1}}\left|a_{1}\right|^{2} R_{\sigma}\left[t-\frac{1}{\beta_{1}}\left(f-f_{1}\right)\right]+\frac{1}{\beta_{2}}\left|a_{2}\right|^{2} R_{\sigma}\left[t-\frac{1}{\beta_{2}}\left(f-f_{2}\right)\right]+\text { cross }- \text { terms. }
$$

where there are two peaks around the auto-terms, deformed by cross terms. As we will see later, these peaks are sufficient for accurate IF estimation.

In the next section we will consider a monocomponent linear FM signal as well as a multi- 
component signal with LFM components to test the IF estimation capabilities and the immunity to noise of the HTD, ETD, WVD and CWD. We also compare these distribution over the SEMG signal with ECG artefact, a patient with malignant ventricular arrhythmia, and a patient with supraventricular arrhythmia.

\section{RESULTS AND DISCUSSION: A COMPARISON OVER BIO- LOGICAL SIGNALS AND NOISY FM SIGNALS}

In this section, we compare the four distributions over normal and abnormal biological signals. Moreover, we study and compare their performance and immunity to noisy signals.

\subsection{A Comparison Over Noisy Linear FM Signals}

The performance of the four distributions over monocomponent linear FM and multicomponent linear FM signals is analyzed.

First, as a monocomponent signal, a linear FM (LFM) test signal $z(t)=a e^{j \phi(t)}, \phi(t)=$ $2 \pi\left(f_{o} t+\beta t^{2} / 2\right)$, with $a=1, f_{o}=0.05 f_{s}, \beta=0.4 f_{s}$ is considered. The instantaneous frequency (IF) is given by $f=\frac{1}{2 \pi} d \phi / d t=f_{o}+\beta t$. For TFD implementation, the signal length $2 N=512$ samples was selected. The sampling frequency was $f_{s}=2 \mathrm{~N} \mathrm{~Hz}$, where the total signal duration was 1 sec. For noise simulation, i.i.d noise samples were added using different SNR's. For each SNR, 1000 Monte Carlo iterations were considered for the purpose of calculating the variance of the IF estimate.

Table 1 shows the result of applying IF estimation on the above noisy LFM for four TFD's. The performance of the HTD and the ETD are distinguished as superior to other TFD's, especially at low SNR's. Performance of the ETD is comparable to that of the HTD for monocomponent signals. It is also evident that the HTD and the ETD surpass other TFD's in robustness where they give the minimum IF variance (called the Cramer-Rao Bound), especially at low SNR's. Note that the bias is nearly zero for all cases.

Second, to test the performance in IF estimation for multicomponent signals, a multicomponent test signal is considered with two linear FM components $z(t)=a_{1} e^{j \phi_{1}(t)}+a_{2} e^{j \phi_{2}(t)}$, $\phi_{1}(t)=2 \pi\left(f_{1} t+\beta_{1} t^{2} / 2\right), \phi_{2}(t)=2 \pi\left(f_{2} t+\beta_{2} t^{2} / 2\right)$, where $a_{1}=a_{2}=1, f_{o 1}=0, f_{o 2}=0.2 f_{s}$, $\beta_{1}=0.45 f_{s}, \beta_{2}=0.3 f_{s}$. The instantaneous frequencies of the individual components are given

respectively by (see [6]) $f_{1}=\frac{1}{2 \pi} d \phi_{1} / d t=f_{o}+\beta_{1} t$ and $f_{2}=\frac{1}{2 \pi} d \phi_{2} / d t=f_{o 2}+\beta_{2} t$. For TFD implementation and robust testing of IF estimation performance, the number of signal points was $2 N=2^{9}$ points, with $f_{s}=2 N \mathrm{~Hz}$ and total signal duration of $1 \mathrm{sec}$. Noise is applied as above. 
Table 1: Performance of various TFD's in IF estimation of a linear FM signal with length $2 N=512$ samples. The sampling frequency was $f_{s}=2 N \mathrm{~Hz}$. For ETD, $\sigma=0.01$, for CWD, $\sigma=19$.

\begin{tabular}{|l|l|l|l|}
\hline SNR (dB) & Var-dB (IF)/ WVD & Var-dB (IF)/ CWD & Var-dB (IF)/ HTD \\
\hline-5 & -24.99 & -35.15 & -59.58 \\
\hline-4 & -27.22 & -48.20 & -60.92 \\
\hline-3 & -33.05 & -49.98 & -61.95 \\
\hline-2 & -47.87 & -51.29 & -62.68 \\
\hline-1 & -62.35 & -52.39 & -63.52 \\
\hline 0 & -63.66 & -53.53 & -64.76 \\
\hline 1 & -64.72 & -54.37 & -65.12 \\
\hline 2 & -65.44 & -55.38 & -66.62 \\
\hline 3 & -66.74 & -56.84 & -67.30 \\
\hline 4 & -67.01 & -57.75 & -67.80 \\
\hline 5 & -68.24 & -59.01 & -68.47 \\
\hline 6 & -68.69 & -59.28 & -69.10 \\
\hline 7 & -70.56 & -60.75 & -70.54 \\
\hline 8 & -71.36 & -61.38 & -72.16 \\
\hline 9 & -71.77 & -62.65 & -73.27 \\
\hline 10 & -74.68 & -63.22 & -75.85 \\
\hline
\end{tabular}

Fig. (6) shows the result of applying IF estimation on the first component of the above noisy multicomponent signal using four TFD's. For each SNR, 1000 Monte Carlo iterations were considered to calculate the variance of the IF estimate. The performance of the ETD is distinguished as superior to other TFD's, including the WVD (which gives ideal concentration for LFM's), especially at low SNR's. The HTD gives a comparable performance, while CWD lags far behind these TFDs. It is worth noting that all TFD's approach the same Cramer-Rao bound as SNR increases.

Figure 6: Performance of different TFD's in IF estimation of the first linear FM component of a multicomponent signal with length $2 N=2^{9}$ samples. The sampling frequency was $f_{s}=2 \mathrm{~N} \mathrm{~Hz}$.

\subsection{A Comparison Over Biological Signals}

In this section we compare the performance of four time-frequency distributions (WVD, CWD, HTD, and ETD) over three types of biological signals: the surface electromyogram (SEMG) with ECG artifacts, the abnormal electrocardiogram (ECG) of a patient with supraventricular Arrhythmia and the abnormal ECG signal of a patient with malignant ventricular arrhythmia. 


\subsubsection{Surface Electromyogram with ECG Artifacts}

As mentioned before, the SEMG signal of the back contains artefact of ECG signals. The removal of such artifacts requires a proper analysis technique to identify the ECG artifacts at time and frequency domain. A SEMG with ECG artifacts data of 1.25 seconds was down-sampled to 200 samples/second. Further, the data was converted by Hilbert transform into its analytical form then processed using the four TFDs.

Fig. (7) shows the time-frequency representations of the SEMG with ECG artifacts in Fig. (1) using HTD, CWD, ETD and WVD. The controlling parameter $\sigma=0.05$ for HTD, 0.015 for ETD, and 19 for CWD. These are practically the optimal values for these TFDs that balance between resolution and cross terms reduction [21]. Any change will compromise one of these factor against the other. Fig. (8) shows the frequency components of this signal at time instant $t=0.7$ second. From these figures we observe that the HTD and the ETD have the best resolution, as they can reveal the frequency components of the signal in Fig. (1) with a resolution much higher than that given by CWD and WVD. The cross-terms in the WVD make the task of identifying the QRS peaks extremely difficult (see Fig. (1)).

Figure 7: Performance comparison and time-frequency representations of the signal in Fig. (1) using HTD, CWD, ETD and WVD. The sampling frequency was $f_{s}=200$ samples/second. For HTD, ETD and CWD $\sigma=0.05,0.015$ and 19 , respectively.

Figure 8: Comparison between the HTD, ETD, CWD and the WVD as related to resolution and crossterm reduction at time instants $t=0.7$ second for the signal in Fig. (1) (with $\sigma=0.05$ for HTD, $\sigma=0.015$ for ETD, $\sigma=19$ for CWD, sampling frequency $=200$ samples $/$ second, and signal length $=1.25$ seconds).

Figs. (9) and (10) show the 3D time-frequency representations of the signal in Fig. (1) using HTD and CWD, respectively. The HTD (Fig. (9)) reveals the two QRS complexes (peaks 'A' and 'C') with a high joint time-frequency resolution, while in Fig. (10) the CWD has broadened these peaks ('A' and 'C') in the time-frequency domain, where the signal features are less clear as compared to those given by the HTD. The HTD, ETD and CWD achieve much better reduction in cross-terms than WVD. However, the HTD outperforms all these distributions in terms of time-frequency resolution in this application.

Fig. (11-up and down) shows the signal magnitude for Fig. (9) as a function of time at time instant $t=0.7$ second and the signal magnitude as a function of frequency at normalized frequency 
Figure 9: 3D time-frequency representations of the signal in Fig. (1) using HTD. The sampling frequency was $f_{s}=200$ samples/second. For HTD, $\sigma=0.05$.

Figure 10: 3D time-frequency representations of the signal in Fig. (1) using CWD. The sampling frequency was $f_{s}=200$ samples/second. For CWD, $\sigma=19$.

$f=0.3$. Fig. (12-up and down) shows the signal magnitude for Fig. (10) as a function of time at time instant $t=0.7$ second and the signal magnitude as a function of frequency at normalized frequency $f=0.3$

Figure 11: Up: signal magnitude for Fig. (9) as a function of time at time instant $t=0.7$ second. Down: signal magnitude as a function of frequency at normalized frequency $f=0.3$.

Figure 12: Up: signal magnitude for Fig. (10) as a function of time at time instant $t=0.7$ second. Down: signal magnitude as a function of frequency at normalized frequency $f=0.3$.

\subsubsection{Abnormal Electrocardiogram of a Patient with Supraventricular Arrhythmia}

A supraventricular rhythm is due to abnormal impulses arising from the atria $[26,27]$. A length of 4.69 seconds of the signal-800 from supraventricular arrhythmia database (Fig. (2)) was converted by Hilbert transform into its analytical forms and processed using the above four TFDs. The sampling frequency for this signal is 128 samples/second. This signal has normal QRS complexes duration of 0.1 second width and a short P-R interval. The QRS complexes have irregular shapes.

Figs. (13), (14) and (15) show the 3D time-frequency representations of the signal in Fig. (2) using HTD, CWD and ETD, respectively. All these techniques are able to detect the three QRS complexes in the signal, however, the ETD, WVD and CWD fail to track changes in the frequency components of the QRS complex of this signal. For example, in Fig. (13) the HTD manage to track the change in the frequency components of each QRS complex as marked by 'A' and 'A1'.

Figure 13: 3D time-frequency representations of the signal in Fig. (2) using HTD. The sampling frequency was $f_{s}=128$ samples/second. For HTD, $\sigma=0.05$. 
Figure 14: 3D time-frequency representations of the signal in Fig. (2) using CWD. The sampling frequency was $f_{s}=128$ samples/ second. For CWD, $\sigma=19$.

Figure 15: 3D time-frequency representations of the signal in Fig. (2) using ETD. The sampling frequency was $f_{s}=128$ samples $/$ second. For ETD, $\sigma=0.015$.

\subsubsection{Abnormal Electrocardiogram of a Patient with Malignant Ventricular Arrhyth- mia}

A length of 2.4 seconds of the signal-418 from malignant ventricular arrhythmia database (Fig. 3) was converted by Hilbert transform into its analytical forms and processed using the four TFDs. The sampling frequency for this signal is 250 samples/second. The QRS complexes in this signal are wide and abnormal, while the T-wave has disappeared. The signal seems to have an irregularity and is changing over time.

Fig. (16) shows the 3D time-frequency representations of the signal in Fig. (3) using the HTD. The superior performance of the HTD is clearly demonstrated in this figure. All frequency components in the signal of Fig. (3) are clearly revealed by the HTD.

Figure 16: 3D time-frequency representations of the signal in Fig. (3) using HTD. The sampling frequency was $f_{s}=250$ samples/second. For HTD, $\sigma=0.05$.

\section{CONCLUSION}

This paper has presented a comparative performance study of four well-known time-frequency distributions (TFDs) in the instantaneous frequency (IF) estimation of biological signals towards identifying normal and abnormal biomedical phenomena. These TFDs are: the Wigner-Ville distribution (WVD), the Choi-Williams distribution (CWD), the Exponential T-distribution (ETD) and the Hyperbolic T-distribution (HTD). Their performance over the normal surface electromyogram (SEMG) with the presence of ECG artifacts and abnormal cardiac signals as well as over mono- and multicomponent FM signals in additive Gaussian noise was compared. When applying IF estimation using the above distributions on the noisy monocomponent linear FM, it was shown that the HTD and the ETD surpass other TFDs in robustness, where they give the minimum variance, especially at low SNRs. In case of multicomponent linear FM, the performance of the 
ETD is distinguished as superior to other TFDs, including the WVD. The HTD gives a comparable performance, while CWD lags far behind these TFDs. In analyzing the SEMG with ECG artifacts, the HTD and the ETD gave the best resolution, as they revealed the whole frequency components of this signal with a resolution much higher than that given by CWD and WVD. The cross terms in the WVD make the task of identifying the QRS peaks extremely difficult. The HTD outperforms all these distributions in terms of time-frequency resolution in this analysis. The high resolution of the HTD in revealing the location in the joint time - frequency plane of the QRS complexes is useful in removing the ECG signal from the SEMG automatically without loss of information.

In case of abnormal signals (a patient with supraventricular arrhythmia and a patient with malignant ventricular arrhythmia), all of the above TFDs are able to detect the QRS complexes in these signals, however, the ETD, WVD and CWD fail to track changes (abnormality) in the frequency components of the QRS complexes of these signals. The HTD has revealed the true structure of the QRS complexes in the supraventricular arrhythmia signal where there are two components with narrow separation in frequency. As such a high resolution time-frequency distribution can be a preprocess towards automatic time-frequency arrhythmias detection and classification.

\section{ACKNOWLEDGEMENT}

This research is supported by a RIF grant from RMIT University, Australia. The authors would like to thank the reviewers for their helpful comments that improved this paper.

\section{References}

[1] C. Bigan and M. S. Woolfson, "Time-frequency analysis of short segments of biomedical data," IEEE Proc.-Sci. Meas. Technol., vol. 147, no. 6, pp. 368-373, Nov. 2000.

[2] R. H. Clayton, A. Murray, "Estimation of the ECG signal spectrum during ventricular fibrillation using the fast Fourier transform and maximum entropy methods," Proceedings of the Computers in Cardiology, pp. 867 - 870, Sept. 1993.

[3] J. C. Wood, D. T. Barry, "Time-frequency analysis of skeletal muscle and cardiac vibrations," Proceedings of the IEEE, vol. 84, issue 9, pp. 1281-1294, Sept. 1996. 
[4] L. Cohen, "Time-frequency distributions - a review" Proceedings of the IEEE, vol. 77, no. 7, pp. 941-981, July 1989.

[5] L. Cohen, Time-Frequency Analysis, Prentice Hall PTR, Englewood Cliffs, New Jersey, 1995.

[6] Z. M. Hussain and B. Boashash, "Adaptive instantaneous frequency estimation of multicomponent FM signals using quadratic time-frequency distributions," IEEE Transactions on Signal Processing, vol. 50, no. 8, pp. 1866 -1876, Aug. 2002.

[7] P. Flandrin, Time-Frequency/ Time-Scale Analysis, Academic Press, Boston, MA, 1998.

[8] L. Qiu and G. Li, "Representation of ECG Signals Based on The Instantaneous Frequency Estimation," Proceedings of ICSP, pp. 1731-1734, 1996.

[9] Z. M. Hussain and B. Boashash, "IF estimation for multicomponent signals," in TimeFrequency Signal Analysis and Processing: A Comprehensive Reference, pp. 437-445, Elsevier, Oxford, UK, 2003.

[10] B. Boashash, Time-Frequency Signal Analysis and Processing: A Comprehensive Reference, Elsevier, Oxford, UK, 2003.

[11] B. Boashash, "Time-frequency signal analysis," in Advances in Spectrum Estimation,, S. Haykin, Ed. Englewood Cliffs, NJ: Prentice-Hall,1991.

[12] Z. M. Hussain and B. Boashash, "Design of time-frequency distributions for amplitude and IF estimation of multicomponent signals," The Sixth International Symposium on Signal Processing and Its Applications (ISSPAŠ2001), 13-16 Aug. 2001.

[13] Physiobank, Physionet, Physiologic signal archives for biomedical research, http://www.physionet.org/physiobank/, viewed Aug. 2005.

[14] P. Celka, B. Boashash, and P. Colditz, "Preprocessing and time-frequency analysis of newborn EEG seizures" IEEE Engineering in Medicine and Biology Magazine, vol. 20, no. 5, pp. 30-39, 2001.

[15] J. R. Cram, G. S. Kasman, and J. Holtz, Introduction to surface electromyography, Gaithersburg, Md.: Aspen Publishers xiv, 408, 1998.

[16] P. A. Parker and R. N. Scott, Myoelectric control of prostheses, Critical Review in Biomedical Engineering, 13(4), pp 283 - 310, 1986. 
[17] S. I. Reger and V. Sahgal, "Analysis of large array surface myoelectric potentials for the low back muscles," the 23rd Annual International Conference of the IEEE Engineering in Medicine and Biology Society, 2001.

[18] S. C. Ragupathy, Classification of static postures using surface electromyogram, Master thesis, RMIT University, 2004.

[19] A. K. Hebbar, and W. J. Hueston, "Management of Common Arrhythmias: Part I. Supraventricular Arrhythmias," Journal of American Family Physician, vol. 65, no. 12, pp. 2479-2486, June 2002.

[20] LJ. Stankovic, V. Katkovnic, "The Wigner distribution of noisy signals with adaptive timefrequency varying window," IEEE Trans. Signal Processing, vol. 47, no. 4, pp. 1099-1108, 1996.

[21] Z. M. Hussain and B. Boashash, "The T-Class of Time-Frequency Distributions: Time-Only Kernels with Amplitude Estimation," to appear in the Journal of the Franklin Institute, 2006.

[22] L. Senhadji, J. J. Bellanger, G. Carrault and J. L. Coatrieux, "Wavelet analysis of ECG signals," in Proc. IEEE/12th Ann. Conf. Eng. Med. Bid. Soc., pp. 811-812, 1990.

[23] I. Daubechies, Ten Lectures on Wavelets, 2nd ed. Philadelphia: SIAM, 1992.

[24] H. Choi, W. Williams, "Improved time-frequency representation of multicomponent signals using exponential kernels," IEEE Trans. Signal Processing, vol. 37, no. 6, pp. 862-871, 1989.

[25] M.A. Malik and J. Saniie, "Performance comparison of time-frequency distributions for ultrasonic nondestructive testing," IEEE Ultrasonics Symposium Proceedings, vol. 1, pp. 701 $704,1996$.

[26] J. A. Luchsinger, J. S. Steinberg, "Resolution of cardiomyopathy after ablation of atrial flutter," J. Am Coll Cardiol, vol. 32, pp. 205-210, 1998.

[27] A. K. Hebbar, and W. J. Hueston, "Management of Common Arrhythmias: Part II. Ventricular Arrhythmias and Arrhythmias in Special Populations," Journal of American Family Physician, vol. 65, no. 12, pp. 2491-2496, June 2002. 